\title{
Application of Close-Range Photogrammetric Technology to Monitor Slope Deformation
}

\author{
Haopeng Huang ${ }^{1,}$, , Jinghui $\mathrm{Liu}^{2, b^{*}}, \mathrm{Hu}_{\mathrm{Li}}^{3, \mathrm{c}}$, Yuan Zhang ${ }^{4, \mathrm{~d}}$, Junshu Gao ${ }^{5, \mathrm{e}}$, \\ Ye $\operatorname{tian}^{6, f}$ \\ 1, 2, 3, 4, 5, ${ }^{6}$ College of Civil Engineering and architecture, China Three Gorges University, P.R.China \\ a179818375@qq.com, byangjianxue33@126.com (corresponding author), '634061434@qq.com, \\ dyuancham@qq.com, ${ }^{\mathrm{e}} 1185030181 @ q q . c o m,{ }^{f} 982574870 @ q q . c o m$
}

Keywords: Slope; Digital close-range photogrammetry; Monitoring; Application.

Abstract. Close-range photogrammetric technology has the advantages of simple equipment, real-time monitoring, high automation level, high precision and non-contact surface measurement. This article is based on a monitoring example of a slope by digital close range photogrammetric technology and compared with the monitoring result by total station. Research shows that close-range photogrammetry can be applied to monitor slope deformation, providing scientific foundations for slope construction and slope design.

\section{Introduction}

Excavation, reinforcement and supporting of slope is an engineering project which is usually involved in the field of civil engineering such as transportation, water conservation and so on. Stability of slope is an issue which engineers focus on most. Deformation monitoring and safety evaluation on slope is the work that engineers must pay attention to. At present there are a lot of methods of monitoring slope stability[1-3]: traditional geodesic method, GPS measurement, measuring robot technology, sensor measurement, but they can only monitor point. When using monitoring point to monitor deformation body, because the number of measuring points is limited, it's difficult to reflect the detail of slope deformation, and feature information is not full-scale enough. It's impossible to obtain other parts or the entire geologic body's mode of spatial displacement. With the rapid development of computer technology and photogrammetric technology[4-6], close-range photogrammetry is a kind of technology that combining coordinates of a few points and abundant information of images, quickly building high-precision digital three-dimensional model of measured object. It has the advantages of operation safety, convenient, high-precision and ample achievements, which is more and more applied to monitor slope deformation.

\section{Digital Photogrammetry}

Close-range photogrammetry is a branch of remote sensing. It is generally accepted that the photogrammetry whose photography distance is less than about 300 meters is called close range photogrammetry. The process of photogrammetry is actually the inverse process of photography, mapping the coordinates from two-dimensional image to three-dimensional world again and restoring the three-dimensional shape of the objects. In order to achieve the process of reconstructing three-dimensional image, according to the binocular stereo vision theory, it usually needs at least two photos with two different angles to complete it. The multi-baseline digital close-range photogrammetry system Lensphoto based on the characteristics of the convergent photography[7-8], breaking the traditional photogrammetry baseline principle, invented the multi-baseline photogrammetry. It solves close-range directional matching and other problem, using the close-range multi-image matching algorithm to collect many points with the same name, then using the close-range aerial triangulation to obtain exterior orientation elements and camera parameters, finally with the multi-ray intersection and block adjustment and free net adjustment, automatically generating the point cloud of three dimensional coordinates and establishing a high precision digital surface model consequently. It 
skillfully solved the incompatible contradiction between the intersection angle, matching and precision in the traditional digital close-range photogrammetry. It fully opened the development space of the close-range technology. The working of the multi-baseline digital close-range photogrammetry system divide into field data collection and indoor data processing. The precision and photographing result of field measurement is the key to obtain high quality measurement results. Common digital camera can be used (the requirements of the pixels is more than 12 million pixels, metric camera can improve the precision of measurement). Generally more than $60 \%$ of the degree of overlap of images is required. After all the field works, indoor work will be done with the data and images, the process of the multi-baseline digital close-range photogrammetry system Lensphoto are shown in Figure 1.

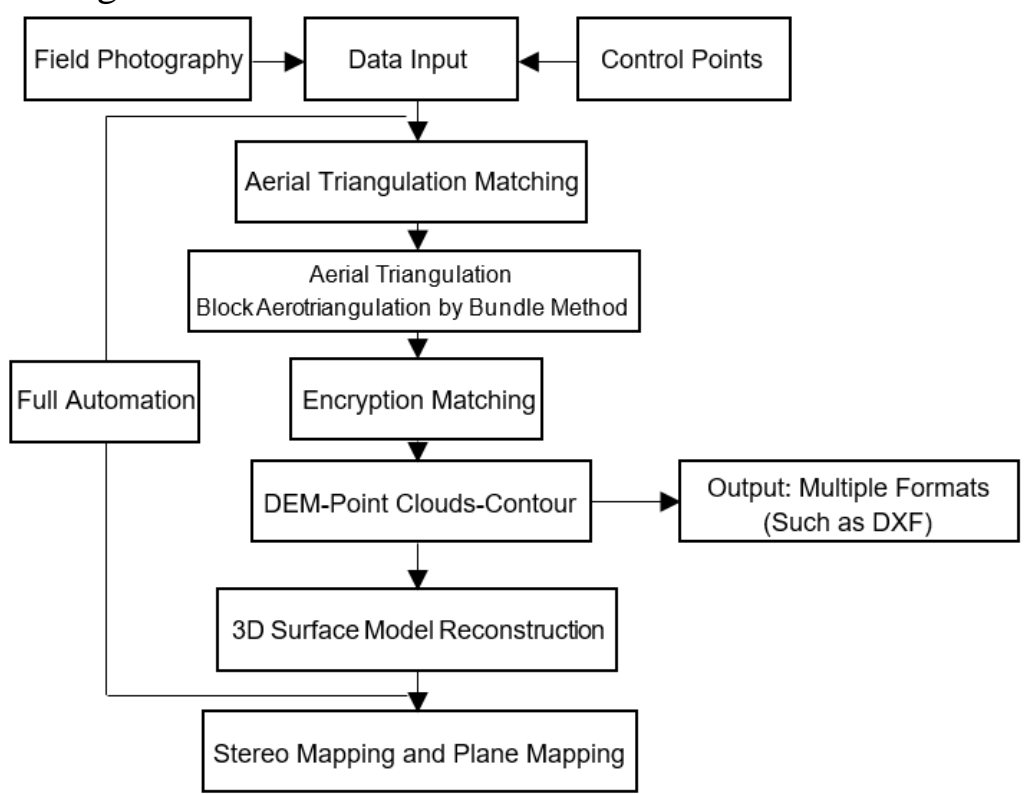

Figure 1 . The process of digital photogrammetry

\section{Application Example of Monitoring Slope Deformation}

\section{Situation of Surveying Area, Image Control Point and Monitoring Points}

The surveying area is located in industrial development zone in Xiangyang. The measure object is the slope protection that is built to safeguard titanium dioxide factory, which is about 10 to 25 meters high and about 200 meters long. The control points were laid out in surrounding of the slop and the monitoring points were laid out in internal slope. Imaging clearly and identifying easily are required for the control points and monitoring points. Material of control points and monitoring points is white-tiled, which is laid in the process of construction built for support. Scene graph of control points is shown in Figure 2.

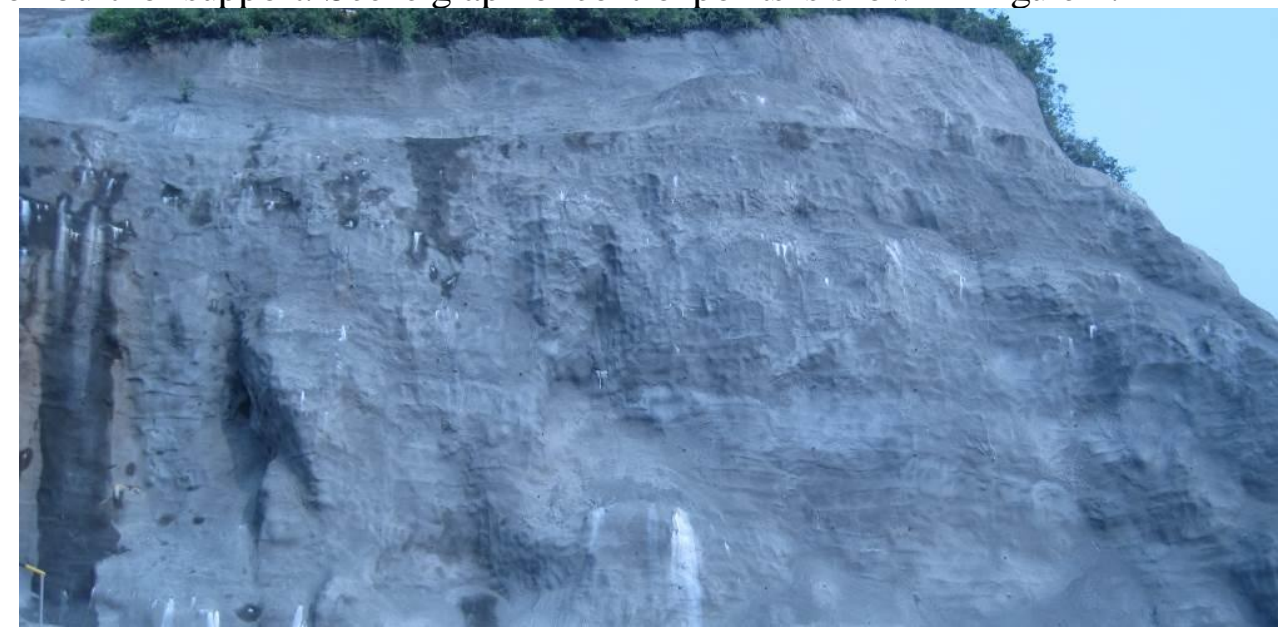

Figure 2. Scene graph of control points 


\section{Deformation monitoring and Data Processing}

Total station and Lensphoto close-range photogrammetry were used to monitor slope deformation, which is convenient for comparison analysis and checking, according to the actual situation of surveying area, Leica TS 30 Total Station with prism and photogrammetry were used at the same control points, measuring the 3D coordinates of all control points. No-prism Total Station and photogrammetry were used at the monitoring points. Digital camera Nikon D100 was used for photogrammetry, which has 12 million pixels and the focal length of $85 \mathrm{~mm}$. The camera was calibrated at the appropriate site[9-11]. According to the length and height of measured slope, in the premise of keeping overlap degree of image, only 6 pictures were needed to cover all the measured slope.There are five camera stations and 30 pictures. The dates of deformation monitoring are shown in Table 1.

Table 1. Dates of Deformation Monitoring

\begin{tabular}{|c|c|c|c|c|c|c|c|c|c|}
\hline \multicolumn{10}{|c|}{ Time Cost } \\
\hline $\begin{array}{l}\text { Measurement at } \\
\text { Image Control Point }\end{array}$ & \multicolumn{2}{|c|}{ Photography } & \multicolumn{2}{|c|}{ Indoor Work } & \multicolumn{2}{|c|}{ Other Work } & \multicolumn{3}{|c|}{ Total Time } \\
\hline $60 \mathrm{~min}$ & \multicolumn{2}{|c|}{$60 \mathrm{~min}$} & \multicolumn{2}{|c|}{$180 \mathrm{~min}$} & \multicolumn{2}{|c|}{$120 \mathrm{~min}$} & \multicolumn{3}{|c|}{$420 \mathrm{~min}$} \\
\hline \multicolumn{10}{|c|}{ Accuracy Statistics of Photogrammetry } \\
\hline Variance of Unit Weight & \multicolumn{5}{|c|}{ Absolute Orientation Accuracy } & \multicolumn{4}{|c|}{ Relative Accuracy } \\
\hline \multirow{2}{*}{$0.001126 \mathrm{~mm}$} & $\operatorname{RmsX}$ & RmsY & Rm: & \multicolumn{2}{|c|}{ Rms } & Plane & \multicolumn{2}{|c|}{ Elevation } & Average \\
\hline & $0.0087 \mathrm{~m}$ & $0.0242 \mathrm{~m}$ & 0.005 & 0.02 & $75 \mathrm{~m}$ & $1 / 12353$ & & 159842 & $1 / 12152$ \\
\hline \multicolumn{10}{|c|}{ Selected Monitoring Points Comparison Analysis } \\
\hline & \multicolumn{5}{|c|}{ Monitoring Point $\mathrm{A}$} & \multicolumn{4}{|c|}{ Monitoring Point B } \\
\hline & $\mathrm{X}$ & & $Y$ & & H & $\mathrm{X}$ & & $\mathrm{Y}$ & $\mathrm{H}$ \\
\hline Total Station $/ \mathrm{m}$ & 3123.244 & & 2.493 & 312 & 7175 & 3009 & 124 & 1123.632 & 215.5683 \\
\hline Photogrammetry/m & 3123.251 & & 2.502 & 312 & 7285 & 3009 & 132 & 1123.641 & 215.5781 \\
\hline Differences/mm & 7.1 & & 5 & & 1 & 8. & & 9.1 & 9.8 \\
\hline
\end{tabular}

Table 1 shows that deformation monitoring results of photogrammetry can achieve centimeter-level and even millimeter-level, which has little difference with results of total station. It's feasible to use close-range photogrammetry in construction of slope monitoring with suitable accuracy. What's more, the implementation and operation of photogrammetry is faster and more convenient than normal geodetic survey, which is safe and reduce the labors, though the accuracy is lower compared with total station monitoring method.

\section{Conclusion}

Based on close-range photogrammetry measuring slope deformation in this paper and using total station method to compare and analyze. Using images photographed at different times, slope deformation can be calculated with the software. It changes the traditional monitoring method to use digital close-range photogrammetry to monitoring the slope stability and achieves fast and high-precision monitoring, greatly reducing the field work, decreasing the labor intensity, enhancing productivity and providing scientific basis to slope treatment and design. With the progress of science and technology, it is expected that the performance and measurement accuracy of close-range photogrammetry monitoring system will be improved on.

\section{References}

[1] Qifeng Yu, Yang Shang. Theory and research of camera measurement[M]. Science Press, 2009, 3.

[2] Xin Xiang, Yanli Wang. Close range photogrammetry application in monitoring of slope deformation[J]. Coal geology of China, 2010, 22(6): 1-3. 
[3] Yunhai Hu. The use of close range photogrammetry technology in landslide monitoring[Master degree thesis][D]. Chengdu: Chengdu University of Technology, 2012.

[4] Changhua Liu. Applications of Digital close range photogrammetry to deformation monitoring of mine[J]. Science of Surveying and Mapping, 2009, 34(4): 197-199.

[5] Peizhen Du. Study on deformation monitoring of buildings based on close range photogrammetry[D]. Ji'nan: Shandong University of Science and Technology. 2007. 25-43.

[6] Cailin Li, Zhang Jianqing, Baoyun Guo. A new method of landslide monitoring based on close range photogrammetry[J]. Engineering and Application of computer. 2011, (03): 6-8.

[7] Zhongshun Sang. Research on close range photogrammetry in tunnel deformation monitoring[D]. Master Thesis of Tongji University, 2008.

[8] Zuxun Zhang, Jianqing Zhang, Tao Ke and so on. Multi baseline Digital Close Range Photogrammetry[J]. Space of Geographic information, 2007, 5(1): 1-4.

[9] Tianzi Li, Hui Guo. Image correction of non-metric digital camera[J]. Surveying and mapping, 2006, (10): 59-61.

[10] Junqian Zhang, Wenjin Wang. A fast planar calibration method for measuring camera[J]. Mapping and spatial geographic information, 2008, (06): 180-186.

[11] Chaohui Yang. Calibration of non-metric digital camera based on linear constraint[J]. Remote sensing information, 2010, (05): 75-79. 